\title{
Hepatic GIP expression is modified by supplementing high-dose thiamine in obese diabetic rats
}

\author{
Yuka Kohda, Akie Maekita, Takao Tanaka, Hitoshi Matsumura \\ Laboratory of Pharmacotherapy, Osaka University of Pharmaceutical Sciences, Japan
}

Obesity is linked with type 2 diabetes in terms of increasing the risk of developing type 2 diabetes and that of its associated morbidity. We previously reported that thiamine supplementation decreases body weight and visceral fat mass in rats with obesity-related diabetes. Glucose-dependent insulinotropic polypeptide (GIP) acts on pancreatic $\beta$ cells to promote insulin secretion. According to established theory, GIP is derived from the gastrointestinal tract. We previously discovered increased expression of the incretin GIP gene (Gip) in the livers of obese rats with diabetes receiving highdose thiamine. We focused on liver-derived GIP to demonstrate GIP protein expression in the liver and visually present localization of GIP in the liver.

Four-week-old male Otsuka Long-Evans Tokushima Fatty (OLETF) rats that exhibit progressive obesity and metabolic disorders, were randomly divided into two groups: an unsupplemented control group and a thiamine-supplemented group receiving $2 \mathrm{~g}$ of thiamine/ $\mathrm{L}$ in drinking water for 51 weeks. Microarray analysis of Gip expression was performed in the livers of OLETF rats at 55 weeks of age. GIP protein expression in the liver was determined by western blotting analysis. Furthermore, GIP was immunohistochemically stained to visualize its localization in the liver.

Gip expression was higher in the livers of high-dose thiamine-supplemented OLETF rats compared with control OLETF rats. At the age of 55 weeks, high-dose thiamine supplemented OLETF rats had increased GIP protein expression compared with control OLETF rats. GIP protein expression was increased in thiamine-supplemented rats despite the suppression of obesity and diabetic complications.

Our previous study demonstrated the increased expression of the Gip in the livers of thiamine-supplemented rats. In addition, we previously reported that the streptozotocin-induced diabetic state triggered GIP expression in rat livers. In the present study, GIP protein expression in the liver was increased in thiamine-supplemented rats compared with that in controls, suggesting that it is involved in preventing and controlling obesity-related diabetic complications. The novel findings of this study that GIP is expressed in the liver, is likely to be added to the story regarding GIP modification of the obese diabetic state. 